Forum 2016 $\cdot 31: 168$

DOI 10.1007/s12312-016-0076-y

C) Springer-Verlag Berlin Heidelberg 2016

Prof. Dr. med. W. Schmiegel

Präsident der Deutschen Krebsgesellschaft e.V.

\title{
Qualität von Tumorboards
}

weise anders aus, als bei einem Board, in dem die internistische Onkologie dominiert. Was das für den einzelnen Betroffenen bedeuten kann, zeigt das Beispiel einer 23 Jahre alten Leberkrebspatientin, die sich kürzlich über Facebook bei der Deutschen Krebsgesellschaft meldete: Die junge Frau erhielt vom ersten Tumorboard die Auskunft, ihr Tumor sei inoperabel, es bliebe nur die palliative Therapie. Weil sie diese Empfehlung nicht akzeptieren wollte, wurde sie bei mehreren anderen Kliniken und Tumorboards vorstellig, bis sie schließlich an einen Chirurgen geriet, der sich doch traute, zu operieren - glücklicherweise mit Erfolg.

Angesichts des exponentiellen Anwachsens therapeutischer Optionen und der zunehmenden Komplexität in der Diagnostik tun wir also gut daran, über die Qualität der Empfehlungen eines Tumorboards nachzudenken. Inwieweit sind dort alle notwendigen Subspezialisierungen, wie in unserem Beispiel die Lebermetastasenchirurgie, abgebildet? Und wie sollte die Qualität der Empfehlungen beurteilt werden? Danach, wie häufig sie sich an medizinischen Leitlinien ausrichten?

Die Umsetzung jeder Leitlinie und Anpassung an den individuellen Fall erfordern immer noch große praktische Erfahrung.

Oder doch eher danach, wie die Board-Empfehlungen den Outcome beim Patienten beeinflussen? Tatsächlich ist die Frage nach geeigneten Qualitätsparametern nicht gut untersucht.

In letzter Konsequenz müssen die ärztlichen Empfehlungen des Tumorboards mit dem Wunsch des Patienten in Einklang gebracht werden

Patientenorientierung und das Treffen gemeinsamer Behandlungsentscheidungen gewinnen im Gesundheitswesen zunehmend an Bedeutung und sind be- sonders in der Krebsversorgung wichtig. Eine gemeinsame Entscheidungsfindung wird zwar von vielen Patienten gewünscht und auf gesundheitspolitischer Ebene gefordert.

\section{I) Bislang fehlt allerdings ein strukturierter Prozess, um den Willen des Patienten verlässlich im Tumorboard abzubilden.}

Das zeigte u.a. eine Untersuchung, die beim Deutschen Krebskongress 2016 vorgestellt wurde. Bessere Kommunikationsfertigkeiten der Ärzte, ein Wandel der ärztlichen Kultur hin zu mehr Patientenbeteiligung, mehr Möglichkeiten für den Patienten, Fragen zu stellen, eine direkte Abfrage der Patientenpräferenz durch die Behandler, all das wünschten sich die Befragten dieser Untersuchung.

Das Mehraugenprinzip der Tumorboards trägt zweifelsohne dazu bei, Fehler $\mathrm{zu}$ vermeiden und Behandlungsoptionen nicht zu übersehen. Auf keinen Fall dürfen wir dieses Prinzip aufgeben. Mit dem Erreichten sollten wir uns allerdings nicht zufrieden geben: Die Frage nach einer hohen Qualität der Tumorboards wird uns sicher in Zukunft beschäftigen.

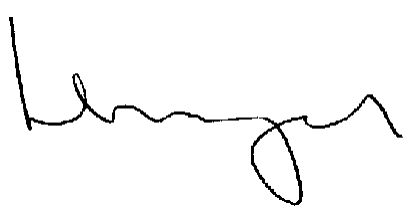

Ihr Wolff Schmiegel

\section{Korrespondenzadresse}

Prof. Dr. med. W. Schmiegel

Ruhr-Universität Bochum,

Medizinische Universitätsklinik

Knappschaftskrankenhaus

44892 Bochum

Wolff.schmiegel@rub.de 